Journal for ImmunoTherapy of Cancer

\title{
Efficacy and safety of vedolizumab and infliximab treatment for immune- mediated diarrhea and colitis in patients with cancer: a two-center observational study
}

Fangwen Zou, ${ }^{1,2}$ David Faleck, ${ }^{3}$ Anusha Thomas, ${ }^{2}$ Jessica Harris, ${ }^{3}$ Deepika Satish, ${ }^{3}$ Xuemei Wang, ${ }^{4}$ Aline Charabaty, ${ }^{5}$ Marc S Ernstoff (i) , ${ }^{6}$ Isabella C Glitza Oliva (1) , Stephen Hanauer, ${ }^{8}$ Jennifer McQuade, ${ }^{7}$ Michel Obeid (1) , ${ }^{9}$ Amishi Shah, ${ }^{10}$ David M Richards, ${ }^{2}$ Elad Sharon (1) , Jedd Wolchok, ${ }^{11}$ John Thompson, ${ }^{12}$ Yinghong Wang (1) ${ }^{2}$

To cite: Zou F, Faleck D, Thomas A, et al. Efficacy and safety of vedolizumab and infliximab treatment for immune-mediated diarrhea and colitis in patients with cancer: a two-center observational study. Journal for ImmunoTherapy of Cancer 2021:9:e003277. doi:10.1136/jitc-2021-003277

- Additional supplemental material is published online only. To view, please visit the journal online (http://dx.doi.org/10. 1136/jitc-2021-003277).

FZ and DF are joint first authors. Accepted 14 October 2021

Check for updates

(C) Author(s) (or their employer(s)) 2021. Re-use permitted under CC BY. Published by BMJ.

For numbered affiliations see end of article.

Correspondence to Dr Yinghong Wang; YWang59@mdanderson.org

\begin{abstract}
Background Current treatment guidelines for immunemediated diarrhea and colitis (IMDC) recommend steroids as first-line therapy, followed by selective immunosuppressive therapy (SIT) (infliximab or vedolizumab) for refractory cases. We aimed to compare the efficacy of these two SITs and their impact on cancer outcomes.

Methods We performed a two-center, retrospective observational cohort study of patients with IMDC who received SITs following steroids from 2016 to 2020. Patients' demographic, clinical, and overall survival data were collected and analyzed.

Results A total of 184 patients (62 vedolizumab, 94 infliximab, 28 combined sequentially) were included. The efficacy of achieving clinical remission of IMDC was similar ( $89 \%$ vs $88 \%, p=0.79$ ) between the two groups. Compared with the infliximab group, the vedolizumab group had a shorter steroid exposure ( 35 vs 50 days, $p<0.001$ ), fewer hospitalizations ( $16 \%$ vs $28 \%, p=0.005$ ), and a shorter hospital stay (median 10.5 vs 13.5 days, $p=0.043$ ), but a longer time to clinical response ( 17.5 vs 13 days, $p=0.012$ ). Longer durations of immune checkpoint inhibitors treatment (OR 1.01, $p=0.004$ ) and steroid use (OR 1.02, $p=0.043$ ), and infliximab use alone (OR 2.51, $p=0.039)$ were associated with higher IMDC recurrence. Furthermore, $\geq 3$ doses of SIT ( $p=0.011$ ), and fewer steroid tapering attempts $(p=0.012)$ were associated with favorable overall survival.

Conclusions Treatment with vedolizumab as compared with infliximab for IMDC led to comparable IMDC response rates, shorter duration of steroid use, fewer hospitalizations, and lower IMDC recurrence, though with slightly longer time to IMDC response. Higher number of SIT doses was associated with better survival outcome, while more steroid exposure resulted in worse patient outcomes.
\end{abstract}

\section{INTRODUCTION}

The advent of immune checkpoint inhibitors (ICIs) has drastically transformed the management of advanced cancers. ${ }^{1}$ However, immune checkpoint blockade can induce broad autoimmunity beyond selective upregulation of tumor-antigen specific T-cells, which can impact other organ systems leading to a variety of immune-related adverse events (irAEs). Immune-mediated diarrhea and colitis (IMDC) is one of the most common irAEs and is often the main reason for ICI discontinuation and morbidities. ${ }^{2}$

Current guidelines for IMDC recommend steroids as first-line treatment, followed by selective immunosuppressive therapy (SIT) for steroid-refractory cases. ${ }^{3}$ However, protracted courses of high-dose steroids can lead to numerous unfavorable complications, and may negatively influence cancer response to ICI therapy and overall cancer prognosis. ${ }^{4}$ Therefore, early introduction of SIT in the course of IMDC to reduce steroid usage and hasten symptom resolution has been proposed as a preferred management strategy. ${ }^{5}$

The two commonly used SITs for IMDC are infliximab (IFX) and vedolizumab (VDZ). IFX is a chimeric antitumor necrosis factor-alpha $(\mathrm{TNF} \alpha)$ monoclonal antibody ${ }^{6}$ that acts by immunosuppression, broadly dampening TNF $\alpha$ 's role in the immune response. In contrast, VDZ is a gut-selective humanized anti- $\alpha_{4} \beta_{7}$ monoclonal antibody that binds to the gut leukocyte adhesion molecule $\alpha_{4} \beta_{7}$-integrin and prevents lymphocyte infiltration in the gut. ${ }^{7}$ Both SITs are approved for treating inflammatory bowel disease (IBD), ${ }^{8}$ and both have shown favorable clinical outcomes in recent small studies of IMDC. ${ }^{910}$ 
Despite the existing data on SITs in treating IMDC, there has not been a direct comparison of the efficacy and safety of these two agents. In addition, the impact of steroids and different SITs on cancer treatment response remains unclear. Therefore, the selection of IFX and/ or VDZ is often based on the clinician's discretion, drug availability, and financial limitation. Given the difference in the underlying mechanisms of action, we hypothesized that VDZ would result in similar efficacy of IMDC treatment, less AEs, and superior cancer outcome and survival.

To address this hypothesis, we conducted a two-center retrospective comparative study of patients with IMDC who received either IFX or/and VDZ after first-line corticosteroid therapy. This study will help determine and compare the clinical efficacy and safety of IFX and VDZ in patients with cancer with IMDC and provide additional reference for clinicians managing IMDC.

\section{METHODS}

\section{Patient cohort}

Patients' informed consent was waived given retrospective design. Included patients were $\geq 18$ years of age that received any ICI and developed IMDC that was treated with steroids and IFX and/or VDZ between March 2016 and March 2020. Patients who had IBD, mesenteric ischemia before ICI treatment, or ICI resumption after IMDC during the study period were excluded. The details of patient distribution are shown in online supplemental table 1. Data collected at Memorial Sloan Kettering Cancer Center were deidentified and centralized at MD Anderson Cancer Center (MDACC) for final analysis.

\section{Clinical data collection}

All patient data were obtained from institutional electronic medical charts and pharmacy databases. Demographic data, cancer and IMDC-related information were extracted. IMDC diagnosis was made by the treating physician based on the exclusion of other etiologies. Complications of immunosuppressants were reported between steroid initiation to 30 days after last dose of any immunosuppressant (steroid or SITs). For patients who received both agents, SITs were given sequentially but not concurrently during the same IMDC event or its recurrence, and the switch was due to treatment failure or AEs. The sequence of administration was at the discretion of treating physicians. Based on the SIT treatments received, patients were divided into three groups for analysis, IFX alone, VDZ alone and both combined sequentially. CTCAE v5.0 $0^{11}$ was used to measure the severity of diarrhea and colitis symptoms. Endoscopic and histological evaluations were also recorded and categorized based on the criteria illustrated previously. ${ }^{12}$

\section{IMDC clinical outcomes assessment}

Clinical outcomes included IMDC-related requirement for multiple hospitalizations or admission to an intensive care unit, duration of hospitalization, clinical remission or response of IMDC, IMDC recurrence, and cancer outcome and OS. IMDC clinical remission was defined as sustained resolution of diarrhea or colitis to grade 1 or lower. IMDC clinical response was defined as a sustained improvement of IMDC symptoms, but not achieving remission. IMDC recurrence was defined as recurrent symptoms beyond 1 month after the initial IMDC episode had resolved. The follow-up duration is administratively censored to maximal 45 months to alleviate the effect of follow-up duration variation among different cohorts. Cancer status was assessed according to the Immunemodified Response Evaluation Criteria in Solid Tumors or oncology note ${ }^{13}$ at IMDC onset and last follow-up.

\section{Statistical analysis}

Statistical analyses were performed by SPSS V.24.0 software (SPSS). Continuous variables were presented as the mean and SD or median and IQR and compared using the Wilcoxon rank-sum test or Kruskal-Wallis test. Categorical variables were described by frequencies and percentages and compared by Fisher's exact and $\chi^{2}$ tests. The Kaplan-Meier method and log-rank test were used to evaluate OS, defined as the duration from IMDC onset to last follow-up or death due to any cause. Univariate and multivariate Cox regression models were fit to assess the association between OS and patient characteristics. Risk factors for IMDC recurrence and cancer progression were assessed by logistic regression models with ORs and 95\% CIs. All statistical tests were two sided, and $\mathrm{p}<0.05$ was considered statistically significant.

\section{RESULTS}

\section{Patient baseline characteristics}

A total of 184 patients (62 VDZ, 94 IFX, 28 combined sequentially) were included (online supplemental table 2). Our cohorts had a median age of 64 years, 118 patients (64\%) were male, and 169 (92\%) were Caucasian. Melanoma (34\%) and genitourinary cancer $(33 \%)$ were the most common malignancies. A total of 153 patients had endoscopic evaluation with confirmed histological inflammation. Fifty-one per cent of patients were treated with IFX alone, 34\% with VDZ alone, and 15\% were treated with combined SIT agents sequentially. The non-gastrointestinal (GI) organ irAEs are summarized in online supplemental table 2. The median follow-up duration was 14 months (IQR 8-27).

\section{Comparison of baseline and IMDC characteristic between IFX and VDZ-treated patients}

With respect to cancer type, the IFX group had a higher proportion of melanoma $(47 \%$ vs $16 \%, \mathrm{p}<0.001)$ and less frequent use of programmed cell death 1 protein/ ligand one inhibitors ( $43 \%$ vs $61 \%, \mathrm{p}=0.041$ ) (table 1 ). In contrast, the VDZ group had a shorter duration of steroid use $(p<0.001)$, fewer steroid tapering attempts $(p=0.016)$, fewer patients with multiple hospitalizations $(\mathrm{p}=0.005)$, and shorter length of cumulative hospital stay $(\mathrm{p}=0.043)$ 
Table 1 Baseline clinical characteristics of patients on treatment of vedolizumab and infliximab alone $(\mathrm{N}=156)$

\begin{tabular}{|c|c|c|c|}
\hline Characteristic & Vedolizumab $n=62$ & Infliximab $n=94$ & $P$ value \\
\hline Median age at IMDC, years (IQR), $n=156$ & $63(49-71)$ & $65(50-73)$ & 0.634 \\
\hline Male sex, no (\%) & $41(66)$ & $58(62)$ & 0.358 \\
\hline Median Charlson Comorbidity Index at colitis diagnosis, points (IQR), n=156 & $8(7-9)$ & $8(6-9)$ & 0.931 \\
\hline Cancer type, no (\%) & & & $<0.001$ \\
\hline Genitourinary cancer & $23(37)$ & $26(28)$ & \\
\hline Lung cancer & $10(16)$ & $16(17)$ & \\
\hline Others & $19(31)$ & $8(8)$ & \\
\hline Cancer stage, no (\%) & & & 0.245 \\
\hline Stage III & $12(19)$ & $14(15)$ & \\
\hline Anti-CTLA-4 & $6(10)$ & 17 (18) & \\
\hline Anti-PD-(L)1 & $38(61)$ & $40(43)$ & \\
\hline Combination & $18(29)$ & $37(39)$ & \\
\hline Median follow-up duration, months (IQR), $n=156$ & $15(11-26)$ & $14(7-30)$ & 0.542 \\
\hline
\end{tabular}

CTLA-4, cytotoxic T-lymphocyte-associated protein 4; IMDC, immune-mediated diarrhea and colitis; PD-(L)-1, programmed cell death 1 protein/ligand.

(table 2). The endoscopic and histologic features are comparable between the two treatment groups. The rate of clinical remission from initial IMDC was equivalent, around $88 \%$, although VDZ group was observed to have a delayed time to clinical response ( 18 vs 13 days, $\mathrm{p}=0.012$ ), and a trend towards longer symptom duration (56 vs 50 days, $\mathrm{p}=0.054$ ) during the initial IMDC episode. Further, we found that, the VDZ alone group had the lowest rate of IMDC recurrence compared with the IFX alone group and patients received combined SITs sequentially (14\% vs $29 \%$ vs $25 \%, \mathrm{p}=0.008$, respectively; online supplemental table 3 ).

\section{Risk factors for IMDC recurrence}

We observed the following risk factors for higher IMDC recurrence (table 3): longer duration of ICI (OR 1.01, $\mathrm{p}=0.004$ ), later onset of IMDC (OR 1.01, $\mathrm{p}=0.002)$, longer duration of steroids (OR 1.02, $\mathrm{p}=0.043$ ), and IFX monotherapy (OR 2.51, $\mathrm{p}=0.039)$. Multivariate logistic regression analysis confirmed that later onset of IMDC after ICI initiation (OR 1.09, $\mathrm{p}=0.002$ ) and longer duration of steroid use (OR 1.48, $\mathrm{p}=0.033$ ) were both risk factors for higher recurrence.

\section{Cancer progression among different treatment regimens and its risk factors}

At the time of IMDC diagnosis, the rate of cancer progression was comparable among all three groups (24\% vs $36 \%$ vs $18 \%, \mathrm{p}=0.107)$. At the time of last follow-up, the rate of cancer progression had increased in all groups, but patients in IFX group had a higher rate compared with the other two groups (54\% vs $34 \%$ vs $43 \%$, respectively, $\mathrm{p}=0.042$, online supplemental figure 1). These findings were further confirmed by a subgroup analysis among 163 patients who had a comparable follow-up time window (2017-2020) (online supplemental table 4).

Univariate analysis showed that higher Charlson Comorbidity Index (CCI) (OR 1.20, p=0.012), longer duration of steroids (OR 1.01, $\mathrm{p}=0.007$ ), and IFX monotherapy (OR 2.32, $\mathrm{p}=0.013$ ) were associated with a higher risk of cancer progression. Multivariate logistic regression analysis confirmed the associations between cancer progression and higher CCI (OR 1.21, p=0.020) and IFX monotherapy (OR 5.24, $\mathrm{p}<0.001$ ). Melanoma-specific cancer type was associated with a lower probability of cancer progression (OR 0.18, p=0.005; online supplemental table 5).

\section{Overall survival analyses}

Patients who received VDZ monotherapy had more favorable OS than those received IFX monotherapy $(p=0.027$, online supplemental figure 2A). Patients who received combined SITs sequentially had an intermediate OS. Fewer steroid tapering attempts were associated with better OS (online supplemental figure 2B, $\mathrm{p}=0.012$ ). Similar outcome was also observed in patients who received $\geq 3$ SIT doses $(\mathrm{p}=0.011$, online supplemental figure 2C). These findings were further confirmed by a subgroup analysis among the 163 patients with a comparable follow-up window (2017-2020) (online supplemental figure 3A). Multivariate Cox regression analysis 
Table 2 IMDC-related characteristics in patients treated with vedolizumab and infliximab alone $(\mathrm{N}=156)$

\begin{tabular}{|c|c|c|c|}
\hline Characteristic & Vedolizumab $n=62$ & Infliximab n=94 & $P$ value \\
\hline Median length of $\mathrm{ICI}$ treatment, days (IQR), $\mathrm{n}=156$ & $73(28-247)$ & $70(28-151)$ & 0.846 \\
\hline Median time from ICI initiation-IMDC onset, days (IQR), $n=156$ & $104(53-212)$ & $96(33-188)$ & 0.065 \\
\hline No of ICI treatments before IMDC, mean (SD) & $7(8)$ & $7(6)$ & 0.235 \\
\hline Diarrhea grade, no (\%) & & & 0.583 \\
\hline $1-2$ & $20(32)$ & $33(35)$ & \\
\hline $3-4$ & $42(68)$ & $61(65)$ & \\
\hline Colitis grade, no (\%) & & & 0.246 \\
\hline $1-2$ & $30(48)$ & $57(61)$ & \\
\hline $3-4$ & $32(52)$ & $37(39)$ & \\
\hline Median duration of initial IMDC symptoms, days (IQR), $n=156$ & $56(27-80)$ & $50(31-69)$ & 0.054 \\
\hline Endoscopy evaluation, no (\%) & $60(96)$ & $65(69)$ & $<0.001$ \\
\hline Endoscopic presentation, no (\%) & & & 0.265 \\
\hline Mucosal ulceration & $17(28)$ & $19(29)$ & \\
\hline Non-ulcerative inflammation & $32(54)$ & $37(57)$ & \\
\hline Normal & $11(18)$ & $9(14)$ & \\
\hline Histology features-no (\%) & & & 0.105 \\
\hline Acute active colitis & $12(20)$ & $24(37)$ & \\
\hline Chronic active colitis & $35(58)$ & $31(48)$ & \\
\hline Microscopic colitis & $13(22)$ & $10(15)$ & \\
\hline IV steroids, no (\%) & $36(58)$ & $58(62)$ & 0.237 \\
\hline Median duration of steroids for initial IMDC, days (IQR), $n=156$ & $35(27-43)$ & $51(41-68)$ & $<0.001$ \\
\hline No of steroid tapering attempts prior to SIT use, median (IQR), $n=156$ & $1(1-3)$ & $2(2-3)$ & 0.016 \\
\hline Median duration from IMDC to first dose of SIT- days (IQR), $n=156$ & $11(9-48)$ & $23(19-37)$ & $<0.001$ \\
\hline No of SIT doses (\%) & & & $<0.001$ \\
\hline $1-2$ & $21(34)$ & $77(82)$ & \\
\hline$\geq 3$ & $41(66)$ & $17(18)$ & \\
\hline Doses of SIT, mean (SD) & $3(2)$ & $2(1)$ & $<0.001$ \\
\hline $\begin{array}{l}\text { Median duration from first dose of SIT to symptom remission or } \\
\text { improvement to grade } 1 \text {, days (IQR), } n=138\end{array}$ & $18(10-40)$ & $13(8-29)$ & 0.012 \\
\hline Hospitalization, no (\%) & $40(65)$ & $67(71)$ & 0.367 \\
\hline Median duration of hospitalization, days (IQR), $\mathrm{n}=107$ & $10(5-15)$ & $14(8-19.8)$ & 0.043 \\
\hline Multiple hospitalizations, no (\%) & $10(16)$ & $26(28)$ & 0.005 \\
\hline Clinical remission, no (\%) & $55(89)$ & $83(88)$ & 0.785 \\
\hline Recurrent IMDC, no. (\%) & $8(14)$ & $27(29)$ & 0.007 \\
\hline Immunosuppressant-associated infection, no (\%) & $12(19)$ & $23(25)$ & 0.184 \\
\hline
\end{tabular}

ICI, immune checkpoint inhibitor; IMDC, immune-mediated diarrhea and colitis; IV, intravenous; SIT, selective immunosuppressive therapy.

demonstrated that higher CCI (HR 1.18, $\mathrm{p}=0.009)$ and IFX monotherapy (HR 2.04, $\mathrm{p}=0.014$ ) were associated with worse OS (online supplemental table 6 ).

In terms of association with different cancer types, superior OS from VDZ among patients with genitourinary and lung cancer was observed when compared with the IFX group ( $\mathrm{p}=0.041$ and $\mathrm{p}=0.046$, respectively, online supplemental figure $4 \mathrm{~A}, \mathrm{~B}$ ). The link between VDZ and longer OS was further confirmed with a subgroup analysis among patients with a similar follow-up window (20172020) (online supplemental figure 3B-D).

\section{AEs related to immunosuppressant treatment}

Infection was one of the most frequent AEs associated with immunosuppressant (steroid and SIT) therapy, accounting for $24 \%$ of all AEs. The infections reported in our study included GI $(41 \%)$ and urinary tract infections (37\%), pneumonia (7\%), periodontitis (7\%), 
Table 3 Univariate and multivariate logistic regression analysis for risk factors for IMDC recurrence

\begin{tabular}{|c|c|c|c|c|c|c|}
\hline \multirow[b]{2}{*}{ Variable } & \multicolumn{3}{|c|}{ Univariate analysis } & \multicolumn{3}{|c|}{ Multivariate analysis } \\
\hline & OR & $95 \% \mathrm{Cl}$ & $P$ value & OR & $95 \% \mathrm{Cl}$ & $\begin{array}{l}P \\
\text { value }\end{array}$ \\
\hline Charlson Comorbidity Index & 1.03 & 0.87 to 1.21 & 0.775 & & & \\
\hline Duration of $\mathrm{ICl}$ treatment & 1.01 & 1.00 to 1.05 & 0.004 & & & \\
\hline \multicolumn{7}{|l|}{ ICI type } \\
\hline Anti-CTLA-4 & & Reference & & & & \\
\hline Anti-PD-(L)-1 & 1.6 & 0.43 to 3.09 & 0.771 & & & \\
\hline Combination & 0.49 & 0.16 to 1.49 & 0.210 & & & \\
\hline Duration from ICI to IMDC onset & 1.09 & 1.03 to 1.15 & 0.002 & 1.09 & 1.03 to 1.15 & 0.002 \\
\hline Duration of colitis symptoms & 1.01 & 0.99 to 1.02 & 0.487 & & & \\
\hline Diarrhea grade $3-4$ vs $1-2$ & 1.06 & 0.51 to 2.21 & 0.879 & & & \\
\hline Colitis grade $3-4$ vs $1-2$ & 0.98 & 0.48 to 1.94 & 0.911 & & & \\
\hline Overall duration of steroids & 1.44 & 1.01 to 2.06 & 0.043 & 1.48 & 1.03 to 2.12 & 0.033 \\
\hline IV steroids & 1.59 & 0.76 to 3.33 & 0.223 & & & \\
\hline \multicolumn{7}{|l|}{ Type of SIT } \\
\hline Vedolizumab & & Reference & & & & \\
\hline Infliximab & 2.51 & 1.05 to 5.99 & 0.039 & & & \\
\hline Both sequentially & 2.86 & 0.89 to 9.19 & 0.078 & & & \\
\hline Dose of SIT & 1.04 & 0.91 to 1.18 & 0.613 & & & \\
\hline Time from IMDC to first dose of SIT & 1.00 & 0.98 to 1.01 & 0.064 & & & \\
\hline
\end{tabular}

CTLA-4, cytotoxic T-lymphocyte-associated protein 4; ICl, immune checkpoint inhibitor; IMDC, immune-mediated diarrhea and colitis; IV, intravenous; PD-(L)-1, programmed cell death 1 protein/ligand; SIT, selective immunosuppressive therapy.

bacteremia (5\%), and others. Overall, the rates of infection were similar between patients who had long and short durations of steroid exposure or received fewer and more steroid treatment courses (online supplemental figure 5A,B). However, the substantial rise of infection was evident in the IFX group with more steroid treatment courses $(19 \%$ vs $39 \%, \mathrm{p}=0.045)$.

\section{DISCUSSION}

This study is the first large, two-center comparative study of the clinical efficacy, AEs and cancer outcomes between IFX and VDZ treatments for patients with IMDC. We observed that patients treated with VDZ as compared with IFX had comparable IMDC response rates, shorter duration of steroid use, fewer hospitalizations, and lower IMDC recurrence, though with slightly longer time to IMDC response.

While IMDC is the second most frequent irAEs, robust evidence for various treatment regimens are lacking, particularly in reference to steroid sparing therapies such as IFX and VDZ. ${ }^{2}$ IFX is more commonly used for IMDC, likely due to several factors. First, as the initial FDA approved SIT in 1998 for IBD, it was also the effective biological agent used early to manage steroid-refractory IMDC. VDZ was not approved for clinical use in IBD until 2014 and therefore is less studied. Furthermore, the advent of biosimilars and large observational cohort studies in IBD showing that an IFX biosimilars has comparable efficacy and safety with the originator and more appealing financial cost could potentially affect the management of IMDC as well. ${ }^{14}{ }^{15}$ Finally, most oncology guidelines recommended VDZ only for patients with contraindications or non-response to IFX, ${ }^{16}$ and so were often not prescribed as first line SIT nor readily covered by insurance. This maybe in part due to the fact that these guidelines are written primarily by oncologists with little experience with anti-integrin therapy.

IFX has been studied extensively in the IBD population and can effectively induce and maintain enteric mucosal healing. ${ }^{17}$ However, given its systemic mode of action, this drug is also associated with serious AEs such as infections and malignancy. ${ }^{18}$ It is important to recognize that the vast majority of studies of IFX provide data on the safety of the long-term TNF-a blockade in a non-cancer population. In the field of IMDC, the limited evidence showing IFX's association with decreased survival comes from the Dutch Melanoma Treatment Registry. ${ }^{19}$ Unlike the broad mechanism of IFX, VDZ can selectively inhibit the interaction between leukocytes and the intestinal vasculature and prevent the influx of inflammatory cells, which mediate the inflammatory process in IMDC. ${ }^{20}$ Given its highly gut-selective mode of action, long-term 
VDZ treatment has a favorable safety profile with low incidence of serious infections and malignancies. ${ }^{21}$ An integrated study on the safety of VDZ based on six IBD trials that involved 2830 patients summarized no increased risk of infections as compared with placebo as well as low rates of malignancies $(<1 \%) .^{22}$

Though there are multiple case series of IFX and VDZ in treating IMDC, ${ }^{10} 23$ our retrospective comparative analysis of these two agents in a cancer population with IMDC is the first of its kind. While both these agents are used routinely in the management of IMDC, it is important to note that most of the available data are extrapolated from clinical trials in patients with IBD. Despite the similarities between IMDC and IBD, there are substantial differences that may impact their relative efficacy and safety, for example, the presence of underlying cancer, and the shorter duration of SIT therapy for IMDC, and underscore the importance of comparative data focused on IMDC.

The majority of our sample was comprised of patients with advanced cancer and severe IMDC. Of note, there were no significant differences in IMDC severity between the SIT treatment groups as assessed by CTCAE grade and endoscopic/histologic findings. Nonetheless, patients treated with IFX received a significantly longer duration of steroid treatment, more steroid treatment courses, and had higher rates of IMDC recurrence as compared with the VDZ treatment group. Together, these observations appear to favor the use VDZ over IFX for treatment of steroid-refractory IMDC, though at the expense of a slightly longer latency to clinical response. The slower response time to VDZ mirrors observations in IBD, though robust data regarding response time are limited. ${ }^{2425}$

However, there were notable differences in prescribing patterns between IFX and VDZ that may have influenced efficacy and clinical outcomes as well. We observed a delay in initiation of IFX as compared with VDZ in the colitis disease course (median of 23 vs 11 days). Prior evidence suggests that early SIT introduction can result in a higher efficacy against IMDC, which may favor the VDZ-treated group. ${ }^{5}$ Moreover, the IFX group received fewer doses of SIT than did the VDZ group, which may have contributed to the significantly higher IMDC recurrence seen with IFX. This observation supports our previous study on the contributing factors associated with lower IMDC recurrence, with higher number of SIT doses correlating with lower recurrence rates. ${ }^{5}$ Some of the outcome differences, therefore, may reflect the wide variances in practice pattern among different providers in terms of SIT choice, timing of SIT initiation as well as doses of SIT administered, rather than differences in the drug mechanism of action alone.

On scrutinizing our subgroup that received combined SITs sequentially, 25 patients $(89 \%)$ switched from IFX to VDZ, while $11 \%$ switched from VDZ to IFX, and $68 \%$ of patients in this group achieved IMDC remission. For this challenging group of patients refractory to one or two lines of SIT, alternative therapies such as $\mathrm{FMT}^{26}$ or other novel agents can be considered. Ustekinumab ${ }^{27}$ and tofacitinib $^{28}$ are employed as induction and maintenance therapy for IBD by inhibition of interleukin 12/23 and Janus Kinase, respectively. These agents require further evaluation in the management of IMDC, and current evidence supporting their use is limited to case series and reports. ${ }^{29-31}$

We observed an overall infection rate of $24 \%$ in our study. These results are in keeping with those from a prior study that noted a significantly lower rate of infections $(30 \%)$ in patients treated with a combination of SIT and steroid compared with long duration of steroid alone $(51 \%) .{ }^{32}$ We suspect that SIT therapy overcame steroid dependence and duration to an extent and potentially negated any significant difference in AEs that might have resulted from prolonged systemic steroids.

We also assessed cancer outcomes among the patients in this study as an exploratory analysis, with the understanding that retrospective, uncontrolled data from a heterogenous group of patients with IMDC poses significant limitations. We observed a significantly higher rate of cancer progression at last follow-up in the IFX-treated group and inferior long-term OS, despite similar baseline cancer status at IMDC onset. While we speculated that considerable exposure to steroids in the IFX group may have contributed to the worse cancer outcomes, this hypothesis was not supported by the outcome of the group who received combined SITs sequentially, which notably received the most intravenous steroids and had equivalent steroid treatment courses. Nonetheless, overall duration of steroid exposure was associated with worse survival in univariate analysis, an observation that has been made in studies of other irAEs as well. ${ }^{33} 34$ Interestingly, when accounting for cumulative doses of SIT treatments, it appeared that more doses were beneficial to patients' overall survival. This observation may reflect the extended colitis disease course in these patients and signs of prolonged ICI effect. This finding is consistent with a previous study showing an association between chronic IMDC and favorable cancer outcome. $^{35}$

Undoubtedly, other confounding factors for example, different tumor biology and burden, differences in ICI regimens, selection of different SIT agent and timing of endoscopy evaluation should also be taken into consideration when interpreting the cancer outcomes and survival data, which we view as hypothesis-generating rather than conclusive data. We are hopeful that the upcoming prospective randomized clinical trial (NCT04407247) between IFX and VDZ to be conducted at MDACC will further delineate the role of each SIT in cancer prognosis. Taking all the pros and cons into consideration, we speculate that a combination strategy of using one dose of IFX for quicker induction followed by long-term VDZ maintenance could potentially minimize the unfavorable effect of IFX and maximize the beneficial aspect of VDZ for both IMDC and cancer outcomes. 
Our study has several limitations. First, this is a retrospective study with all the inherent limitations that stem from this study design. Second, because the study is from two tertiary cancer centers, results may not be generally applicable to all types of clinical practice. Third, while this study is the largest regarding IMDC and SITs, the sample size in the combined group was relatively small, which limited the power in the subgroup analysis. Fourth, the limited data availability in retrospective chart review prohibited the accurate collection of cumulative dose and duration of steroid use through all IMDC episodes, and additional cancer therapy after ICI termination, which could potentially confound the results of cancer outcome and survival. Fifth, given the exclusion of patients treated with steroids alone, the specific impact of SIT on cancer outcome and OS could not be assessed. Sixth, we note a significant difference in cancer type, and ICI regimens among the two treatment groups and acknowledge that tumor biology, and different mechanism of action from specific ICI agents may play a role in both irAEs as well as cancer outcomes. Last, given the lack of standard guidelines for SITs use for IMDC, there were significant variations in the treatment regimens utilized by the treating physicians, which may have introduced biases to the study.

In conclusion, in a large retrospective two-center cohort, we observed that treatment with VDZ compared with IFX for IMDC led to comparable rates of colitis clinical remission, with shorter duration of steroid use, fewer hospitalizations and lower rate of IMDC recurrence, though with slower onset of action. Furthermore, higher doses of SIT were associated with better survival outcome, while more steroid exposure resulted in worse patient outcome. Future prospective randomized trials are needed to further confirm these observations and clarify the impact of different SITs on cancer outcome.

\section{Author affiliations}

1Department of Oncology, Second Xiangya Hospital, Changsha, Hunan, China ${ }^{2}$ Department of Gastroenterology, Hepatology \& Nutrition, The University of Texas MD Anderson Cancer Center, Houston, Texas, USA

${ }^{3}$ Department of Gastroenterology, Hepatology and Nutrition, Memorial Sloan Kettering Cancer Center, New York, New York, USA

${ }^{4}$ Department of Biostatistics, The University of Texas MD Anderson Cancer Center, Houston, Texas, USA

${ }^{5}$ Department of Gastroenterology, Johns Hopkins University, Washington, DC, USA ${ }^{6}$ Division of Cancer Treatment and Diagnosis, National Cancer Institute, Bethesda, Maryland, USA

${ }^{7}$ Department of Melanoma Medical Oncology, The University of Texas MD Anderson Cancer Center, Houston, Texas, USA

${ }^{8}$ Department of Medicine, Northwestern University, Chicago, Illinois, USA

${ }^{9}$ Department of Medicine, Centre Hospitalier Universitaire Vaudois, Lausanne, Switzerland

${ }^{10}$ Department of Genitourinary Medical Oncology, The University of Texas MD Anderson Cancer Center, Houston, Texas, USA

${ }^{11}$ Human Oncology and Pathogenesis Program, Department of Medicine, Memorial Sloan Kettering Cancer Center, New York, New York, USA

${ }^{12}$ University of Washington, Seattle Cancer Care Alliance, Fred Hutchinson Cancer Research Center, Seattle, Washington, USA

\section{Twitter Isabella C Glitza Oliva @GlitzaMDPhD and Jennifer McQuade @} McQuadeMDLAc

Contributors YW was the senior author of this study and also guarantor, developed the concept, designed the study, interpreted the results, ensured that the accuracy and integrity of the data was preserved at all stages, agreed to be accountable for all aspects of this study, accepts full responsibility for the work and/or the conduct of the study, had access to the data and controlled the decision to publish, was in charge of the overall direction and planning of the study, and contributed to the writing of the manuscript with input from all authors. FZ and DF collected the data for the study, conducted and interpreted the analysis, and wrote the manuscript. $\mathrm{JH}$ and DS collected the data for the study and edited the paper. XW conducted biostatistical analysis. AT, AC, MSE, IG, SH, JM, MO, AS, DMR, ES, JW and JT revised critically the final version of the manuscript. All authors read and approved the final manuscript.

Funding The authors have not declared a specific grant for this research from any funding agency in the public, commercial or not-for-profit sectors.

Competing interests No, there are no competing interests.

Patient consent for publication Not applicable.

Ethics approval This retrospective, case-control, observational, two-center study was approved by the institutional review board at MD Anderson Cancer Center (MDACC) and Memorial Sloan Kettering Cancer Center (MSKCC).

Provenance and peer review Not commissioned; externally peer reviewed.

Data availability statement Data are available on reasonable request.

Supplemental material This content has been supplied by the author(s). It has not been vetted by BMJ Publishing Group Limited (BMJ) and may not have been peer-reviewed. Any opinions or recommendations discussed are solely those of the author(s) and are not endorsed by BMJ. BMJ disclaims all liability and responsibility arising from any reliance placed on the content. Where the content includes any translated material, BMJ does not warrant the accuracy and reliability of the translations (including but not limited to local regulations, clinical guidelines, terminology, drug names and drug dosages), and is not responsible for any error and/or omissions arising from translation and adaptation or otherwise.

Open access This is an open access article distributed in accordance with the Creative Commons Attribution 4.0 Unported (CC BY 4.0) license, which permits others to copy, redistribute, remix, transform and build upon this work for any purpose, provided the original work is properly cited, a link to the licence is given, and indication of whether changes were made. See https://creativecommons.org/ licenses/by/4.0/.

Author note Writing Assistance: Medical editing of this paper was provided by Bryan Tutt, Scientific Editor, Research Medical Library, at MD Anderson Cancer Center.

\section{ORCID iDs}

Marc S Ernstoff http://orcid.org/0000-0002-8132-7069

Isabella C Glitza Oliva http://orcid.org/0000-0002-4530-7765

Michel Obeid http://orcid.org/0000-0003-2095-2677

Elad Sharon http://orcid.org/0000-0002-0044-9719

Yinghong Wang http://orcid.org/0000-0002-5148-6130

\section{REFERENCES}

1 Dijkstra KK, Voabil P, Schumacher TN, et al. Genomics- and transcriptomics-based patient selection for cancer treatment with immune checkpoint inhibitors: a review. JAMA Oncol 2016;2:1490-5.

2 Wang DY, Salem J-E, Cohen JV, et al. Fatal toxic effects associated with immune checkpoint inhibitors: a systematic review and metaanalysis. JAMA Oncol 2018;4:1721-8.

3 Abu-Sbeih H, Ali FS, Naqash AR, et al. Resumption of immune checkpoint inhibitor therapy after immune-mediated colitis. J Clin Oncol 2019;37:2738-45.

4 Grennan D, Wang S. Steroid side effects. JAMA 2019;322:282.

5 Abu-Sbeih H, Ali FS, Wang X, et al. Early introduction of selective immunosuppressive therapy associated with favorable clinical outcomes in patients with immune checkpoint inhibitor-induced colitis. J Immunother Cancer 2019;7:93.

6 Friedman CF, Proverbs-Singh TA, Postow MA. Treatment of the immune-related adverse effects of immune checkpoint inhibitors: a review. JAMA Oncol 2016;2:1346-53.

7 Novak G, Hindryckx P, Khanna R, et al. The safety of vedolizumab for the treatment of ulcerative colitis. Expert Opin Drug Saf 2017;16:501-7.

8 Wright EK, Ding NS, Niewiadomski O. Management of inflammatory bowel disease. Med J Aust 2018;209:318-23.

9 Johnson $\mathrm{DH}$, Zobniw CM, Trinh VA, et al. Infliximab associated with faster symptom resolution compared with corticosteroids alone for 
the management of immune-related enterocolitis. J Immunother Cancer 2018;6:103.

10 Abu-Sbeih H, Ali FS, Alsaadi D, et al. Outcomes of vedolizumab therapy in patients with immune checkpoint inhibitor-induced colitis: a multi-center study. J Immunother Cancer 2018;6:142.

11 Johnson DB, Chandra S, Sosman JA. Immune checkpoint inhibitor toxicity in 2018. JAMA 2018;320:1702-3.

12 Abu-Sbeih H, Ali FS, Luo W, et al. Importance of endoscopic and histological evaluation in the management of immune checkpoint inhibitor-induced colitis. J Immunother Cancer 2018;6:95.

13 Hodi FS, Ballinger M, Lyons B, et al. Immune-modified response evaluation criteria in solid tumors (imRECIST): refining guidelines to assess the clinical benefit of cancer immunotherapy. J Clin Oncol 2018;36:850-8.

14 Thompson JA. New NCCN guidelines: recognition and management of Immunotherapy-Related toxicity. J Natl Compr Canc Netw 2018;16:594-6.

15 Brahmer JR, Lacchetti C, Schneider BJ, et al. Management of immune-related adverse events in patients treated with immune checkpoint inhibitor therapy: American Society of Clinical Oncology Clinical Practice Guideline. J Clin Oncol 2018;36:1714-68.

16 Singh BP, Marshall JL, He AR. Workup and management of immune-mediated colitis in patients treated with immune checkpoint inhibitors. Oncologist 2020;25:197-202.

17 Hemperly A, Vande Casteele N. Clinical pharmacokinetics and pharmacodynamics of infliximab in the treatment of inflammatory bowel disease. Clin Pharmacokinet 2018;57:929-42.

18 Hanauer SB, Feagan BG, Lichtenstein GR, et al. Maintenance infliximab for Crohn's disease: the ACCENT I randomised trial. Lancet 2002;359:1541-9.

19 Verheijden RJ, May AM, Blank CU, et al. Association of anti-TNF with decreased survival in steroid refractory ipilimumab and antiPD1-treated patients in the Dutch melanoma treatment registry. Clin Cancer Res 2020;26:2268-74.

20 Sands BE, Peyrin-Biroulet L, Loftus EV, et al. Vedolizumab versus adalimumab for moderate-to-severe ulcerative colitis. N Engl J Med 2019;381:1215-26.

21 Colombel J-F, Colombel J-F, Feagan BG, et al. Long-term efficacy of Vedolizumab for ulcerative colitis. J Crohns Colitis 2017;11:400-11.

22 Colombel J-F, Sands BE, Rutgeerts P, et al. The safety of vedolizumab for ulcerative colitis and Crohn's disease. Gut 2017;66:839-51.
23 Johnston RL, Lutzky J, Chodhry A, et al. Cytotoxic T-lymphocyteassociated antigen 4 antibody-induced colitis and its management with infliximab. Dig Dis Sci 2009;54:2538-40.

24 Feagan BG, Sandborn WJ, Colombel J-F, et al. Incidence of Arthritis/ Arthralgia in inflammatory bowel disease with long-term Vedolizumab treatment: post hoc analyses of the gemini trials. J Crohns Colitis 2019;13:50-7.

25 Feagan BG, Lasch K, Lissoos T, et al. Rapid response to vedolizumab therapy in biologic-naive patients with inflammatory bowel diseases. Clin Gastroenterol Hepatol 2019;17:130-8.

26 Wang Y, Wiesnoski DH, Helmink BA, et al. Fecal microbiota transplantation for refractory immune checkpoint inhibitor-associated colitis. Nat Med 2018;24:1804-8.

27 Feagan BG, Sandborn WJ, Gasink C, et al. Ustekinumab as induction and maintenance therapy for Crohn's disease. $N$ Engl J Med 2016;375:1946-60.

28 Sandborn WJ, Su C, Sands BE, et al. Tofacitinib as induction and maintenance therapy for ulcerative colitis. N Engl J Med 2017;376:1723-36.

29 Esfahani K, Hudson M, Batist G. Tofacitinib for refractory immunerelated colitis from PD-1 therapy. N Engl J Med 2020;382:2374-5.

30 Bishu S, Melia J, Sharfman W, et al. Efficacy and outcome of tofacitinib in immune checkpoint inhibitor colitis. Gastroenterology 2021;160:932-4.

31 Thomas AS, Ma W, Wang Y. Ustekinumab for refractory colitis associated with immune checkpoint inhibitors. $N$ Engl $\mathrm{J}$ Med 2021;384:581-3.

32 Abu-Sbein H, Tang T, Ali FS, et al. The impact of immune checkpoint inhibitor-related adverse events and their immunosuppressive treatment on patients' outcomes. J Immunother Precis Oncol 2018;1:7-18.

33 Faje AT, Lawrence D, Flaherty K, et al. High-dose glucocorticoids for the treatment of ipilimumab-induced hypophysitis is associated with reduced survival in patients with melanoma. Cancer 2018;124:3706-14.

34 Bai X, Hu J, Betof Warner A, et al. Early use of high-dose glucocorticoid for the management of irAE is associated with poorer survival in patients with advanced melanoma treated with anti-PD-1 monotherapy. Clin Cancer Res 2021:clincanres.1283.

35 Zou F, Abu-Sbeih H, Ma W, et al. Association of chronic immunemediated diarrhea and colitis with favorable cancer response. J Natl Compr Canc Netw 2020;19:1-9. 